Adaptation of New Colombian Food-based Complementary Feeding Recommendations using Linear Programming

Marion Tharrey $(\mathrm{MS})^{1}$, Gilma A Olaya $(\mathrm{PhD})^{2,3}$, Mary Fewtrell $(\mathrm{MD})^{2}$, Elaine Ferguson $(\mathrm{PhD})^{1}$

${ }^{1}$ Department of Population Health, London School of Hygiene and Tropical Medicine, London, UK;

${ }^{2}$ Childhood Nutrition Research Centre, UCL GOS Institute of Child Health, London, UK;

${ }^{3}$ Nutrition and Biochemistry Department, Faculty of Sciences, Pontificia Universidad Javeriana, Bogota, Colombia

Corresponding Author: Elaine Ferguson, London School of Hygiene and

Tropical Medicine, Keppel Street, London WC1E 7HT, UK

Email: elaine.ferguson@1shtm.ac.uk 


\begin{abstract}
Objective: To use Linear Programming (LP) analyses to adapt New Complementary Feeding Guidelines (NCFg) designed for infants aged 6-12 month (m) living in poor socioeconomic circumstances in Bogota to ensure dietary adequacy for young children aged 12-23 m.

Design: A secondary data analysis was performed using dietary and anthropometric data collected from 12 m old infants $(n=72)$ participating in a randomised controlled trial. LP analyses were performed to identify nutrients whose requirements were difficult to achieve using local foods as consumed; and to test and compare the NCFg and alternative food-based recommendations (FBRs) on the basis of dietary adequacy, for 11 micronutrients, at the population level.
\end{abstract}

Results: Thiamine recommended nutrient intakes for these young children could not be achieved given local foods as consumed. NCFg focusing only on meat, fruits, vegetables and breast milk ensured dietary adequacy at the population level for only 4 micronutrients, increasing to 8/11 modelled micronutrients when the FBRs promoted legumes, dairy, vitaminA rich vegetables and chicken giblets. None of the FBRs tested ensured population-level dietary adequacy for thiamine, niacin and iron unless a fortified infant food was recommended.

Conclusion: This study demonstrated the value of using LP to adapt NCFg for a different age group than the one for which they were designed. Our analyses suggest that to ensure dietary adequacy for 12-23 m olds these adaptions should include legumes, dairy products, vitamin A rich vegetables, organ meat and a fortified food.

Keywords: nutritional adequacy; dietary practices; micronutrients; food based recommendations; young children 
What is Known/What is New:

\section{What is Known}

Complementary feeding diets fed to young children living in low and middle income countries are often low in essential micronutrients because they include a limited number of foods

A method based on linear programming analyses has been developed to formulate realistic population-specific food-based recommendations for addressing under-nutrition

\section{What is New}

In this study we demonstrate how this method can be used to adapt an existing set of foodbased recommendations for 6-12 m old infants in Colombia, for slightly older population (12$23 \mathrm{~m}$ old), to ensure dietary adequacy 


\section{Introduction}

Adequate nutrition during the first 2 years of life is important to ensure healthy growth and development $(1,2)$. To prevent nutrient deficiencies and their associated negative functional consequences, the Global Strategy for Infant and Young Child Feeding (3) recommends the introduction of a nutritionally adequate and safe complementary feeding diet from $6 \mathrm{~m}$ of age, which is high in iron and zinc (1-5). In Colombia, despite efforts to improve infant and young child feeding practices, micronutrient deficiencies have increased (6-8). A possible contributory factor has been the use of complementary feeding guidelines that lack specific advice in terms of food type, preparation and serving size. In response, New Complementary Feeding Guidelines (NCFg) were developed for 6-12 m old infants living in low socioeconomic conditions in Bogota, with an emphasis on using locally available, acceptable and affordable foods and red meat, including chicken liver, as a source of iron and zinc (9). These recommendations were shown to be effective for improving feeding practices and infant haemoglobin levels in a randomised control trial (RCT) of infants from 6 to $12 \mathrm{~m}$ of age (10). However, questions remain about whether the NCFg would ensure overall dietary adequacy for a slightly older population (12-23 m old).

A novel approach, for developing and testing population-specific food-based recommendations (FBRs) is to use linear programming (LP) analysis $(11,12)$. To facilitate its use, the software programme - Optifood - was recently developed (13), which identifies nutrient gaps that cannot be filled using local foods as currently consumed and objectively identifies optimized combinations of local foods that will meet or come as close as possible to meeting the nutrient needs of a specific target group (13). Thus the aim of this study was to use LP analyses to identify nutrient gaps in local complementary feeding diets (i.e., "problem nutrients"), evaluate the extent to which the recently developed NCFg would ensure dietary adequacy for young children (12-23 m old), and determine how the NCFg could be adapted to 
ensure dietary adequacy across this age range $(14,15)$. 


\section{Subjects and Methods}

\section{Study design}

A secondary analysis was performed using dietary and anthropometric data collected from 72 infants aged $12 \mathrm{~m}$ old participating in a randomised control trial, in Bogota, Colombia from August 2010 to 2011. Trial design and data collection have been described in detail elsewhere (9). Briefly, full term healthy infants who were part of the growth-monitoring programme in Suba and Fontibon hospitals in Bogota, Colombia (n=85) were purposefully selected to participate in the trial. Dietary intake data were collected via a 24-h recall at the end of the intervention period when the infants were $12 \mathrm{~m}$ of age. Mothers described all foods consumed by the infant in the last 24 hours. To estimate food portion sizes, the amount of each food (as tablespoons, cups, etc) was manually converted into grams consumed per day based on standardized measures previously calculated (10). Energy and nutrient intakes were estimated using information on the nutrient content of 208 foods in the Colombian Food Composition Tables, USDA National Nutrient Database for Standard Reference, food labels (infant food) and manufacturers' information for formula milk (16). Daily breast milk serving sizes were estimated, for each infant, using an algorithm based on published data (17), and taking into account our previous unpublished data and the information recorded in the 24 hour recall on (i) number of breastfeeds/day, (ii) duration of each breast feed, (iii) number of breastfeeds per night and (iv) infant appetite. Nutrient intakes from breast milk were estimated using Colombian Food Composition Table data, assuming $70 \mathrm{Kcal} / 100 \mathrm{ml}$ for energy.

The dietary data and the infants' mean body weight were used to generate the model parameters for the LP analyses. All LP analyses were performed using the software programme - Optifood (13). 
Ethical approval for the RCT was obtained from the research ethics committees at University College London and Pontificia Universidad Javeriana. The trial was registered at http://isrctn.org as ISRCTN57733004.

\section{Data Analyses}

\section{Preparation of Optifood model parameters}

Dietary data collected from both the intervention and control groups were pooled and used to define the model parameters. This process was justified because the dietary intake data are only used as a guide to define model parameters that reflect feasible practice. From the 105 foods reported in the 24-hour recalls, 2 simplified food lists were created (Supplemental Digital Table 1). In the first, 30 foods were excluded to model 75 foods in the series 1 and 2 analyses; and in the second, the commercial fortified food products consumed by the infants were added to the first food list to model 77 foods. The model constraints, which were used to ensure realistic modelled 7-day diets, were inequality constraints based on the minimum and maximum number of daily servings per week for foods, food groups and food sub-groups consumed by the infants, as well as an equality constraint on the 7-day diet energy content (Supplemental Digital Table 1). Daily servings per week $>7$ would mean that at least two different types of foods from that specific food group or sub-group were selected in the modelled 7-day diet.

The main NCFg recommendations, covering the period from 6 to $12 \mathrm{~m}$ of age, were 1 ) feed your infant meat $\geq 5 \mathrm{~d} / \mathrm{wk}$, including red meat (beef, organ meat, especially chicken liver) $\geq 3$ $\mathrm{d} / \mathrm{wk}, 2$ ) continue breastfeeding until 2 y of age, 3) feed your infant fruits and vegetables every day, 4) introduce legumes and eggs at $10 \mathrm{~m}$ of age, and 5) feed your infant dairy products from $11 \mathrm{~m}$ of age. Thus, for $12 \mathrm{~m}$ old infants, the model constraints, for each individual NCFg recommendation tested, were: $\geq 5$ servings/w of meat; $\geq 3$ servings/w of red 
meat; $\geq 1$ serving/w of cows' liver; $\geq 1$ serving/w of chicken giblets (liver and heart), $\geq 7$ servings/w of fruits, $\geq 7$ servings/w of vegetables, $\geq 2$ servings/w of legumes, $\geq 7$ servings/w of eggs and $\geq 14$ servings/w of dairy products. The number of servings/w modelled for legumes, eggs and dairy products corresponded to the reported median number of servings/w consumed by the $12 \mathrm{~m}$ old infants in our study.

\section{Linear Programming Analyses}

Three series of analyses were performed (Table 1), using the software programme - Optifood (13). In the first series of analyses, the NCFg were evaluated by testing each of its nine individual FBR (see above) and all combinations of these individual FBRs (Module III worst case scenario analyses) to identify the best combination of individual FBRs for the $\mathrm{NCFg}$. The best combination was defined as the one with the lowest number of combined individual FBRs that, when tested, had the highest number of minimised nutrient values $\geq 70 \%$ of their RNIs.

In the second series of analyses, the "problem nutrients" were identified as those whose requirements could not be met using any realistic combination of foods in a 7-day diet (i.e., Module III - best case scenario analyses). FBRs that would improve the NCFg, for 12-23 m old children, were identified using the food patterns of the Module II - nutritionally best diet (i.e., the diet that came as close as possible to achieving $100 \%$ of the RNIs for all nutrients modelled). These FBRs were tested and compared to select the best set of individual FBRs to guide refinements of the NCFg (Module III - worst case scenario analyses).

In the third series of analyses, fortified infant food products were evaluated (Module III; model constraint on fortified products was $\geq 2$ servings/w). These products, which were consumed by the infants in the trial, were commercially available fortified infant cereals and a fortified flour Bienesterina $@$ produced by the Colombian Family Welfare Institute (a mix of 
wheat flour, soya flour and skimmed milk powder) (18).

Two sensitivity analyses were also performed to determine whether the results were sensitive to the RNI selected for iron, and the daily amounts of BM modelled. For iron, we changed its WHO/FAO RNI from medium bioavailability (10\%) to high bioavailability (15\%). For BM, we decreased the amount of BM modelled from $483 \mathrm{~g} / \mathrm{d}$ to a low BM intake, for a $12-23 \mathrm{~m}$ old child, of $175 \mathrm{~g} / \mathrm{d}(19)$. 


\section{RESULTS}

\section{The Model parameters}

Characteristics of infants at $12 \mathrm{~m}$ are given in Table 2. The mean body weight of the study population was $8.98 \mathrm{~kg}$ resulting in a calculated energy requirement of $741 \mathrm{kcal} / \mathrm{d}$ (20) to define the models' energy constraint (equality constraint). The food list, median daily serving sizes and all food pattern model constraints used in the analyses are shown in online supplementary material (Supplemental Digital Table 2).

\section{Series one analyses: Evaluation of the NCFg for young children aged 12-23m}

The NCFg recommendations would ensure dietary adequacy for 12-23 m old children (at the population level) for 7 of the 11 micronutrients modelled but thiamine, niacin, folate and iron remained below 70\% RNI (Module III - worst case scenario analyses). Meat and red meat (but not organ meat) appeared to be optional, because excluding them from the $\mathrm{NCFg}$ recommendations did not affect the number of micronutrients achieving dietary adequacy (Table 3 and Supplemental Digital Table 3). Eggs and dairy products alone ensured adequacy for 6 of 11 micronutrients.

Series two analyses: The "problem nutrients" and adaption of the NCFg for use in young children aged 12-23m

\section{Problem Nutrients}

Thiamine was the only "problem nutrient" for this population of $12 \mathrm{~m}$ old children, given that the maximised thiamine content of the diet was only $92 \%$ of its RNI in the Module III - best case scenario analyses. For all other nutrients the maximised diets achieved or exceeded $100 \%$ of their RNIs. 
How to optimize the nutritional adequacy of NCFg for use in young children aged 12-23m

Based on the nutritionally best diet formulated in Module II, which provided $100 \%$ of the RNIs for all nutrients except thiamine, we identified eight key FBRs: 21 servings/w of vegetables of which 14 servings/w are vitamin A rich vegetables; 7 servings/w of vitamin $\mathrm{C}$ rich fruit; 7 servings/w of legumes; 4 servings/w of organ meat of which $\geq 3$ servings/w are chicken giblets; 14 servings/w of dairy products of which $\geq 7$ servings/w are cows' milk. These eight FBRs were systematically tested (Module III) to identify the best set for improving the NCFg for use in young children. The results showed dietary adequacy was achieved for all nutrients except thiamine, niacin and iron; which remained at less than 50\% of their RNIs in all Module III - worst case scenario analyses (Table 4). Of the improved FBRs, chicken giblets (3 servings/w) was the most important FBR for ensuring dietary adequacy at the population level; and when combined with vitamin A-rich vegetables (14 servings/w), legumes (7 servings/w), dairy products (14 servings/w) and milk (7 servings/w) ensured dietary adequacy for at least eight of the 11 micronutrients modelled. The full sets of analyses results are shown in the Supplemental Digital Table 4.

The final set of FBRs selected to include in the NCFg adapted for use in young children was:

- 14 servings/w of vitamin-A rich vegetables;

- 7 servings/w of legumes;

- 14 servings/w of dairy products of which $\geq 7$ servings/w are cow's milk;

- 3 servings/w of chicken giblets;

This set of FBRs ensured population level dietary adequacy for at least eight nutrients; and the minimised $\%$ RNIs for thiamine, niacin and iron were $>40 \%$.

\section{Series three analyses: Fortified foods in the FBRs}


The commercial fortified foods consumed by $12 \mathrm{~m}$ old infants in this study were fortified infant cereals (13\% of infants) and fortified flour ( $7 \%$ of infants). The results showed that a set of FBRs that included fortified foods ( 7 servings/w) accompanied by dairy products, vitamin A-rich vegetables, legumes and organ meat would ensure the daily requirements for all nutrients were met (Table 4; full set of analyses in Supplemental Digital Table 5).

\section{Sensitivity to BM intake and the RNI selected for iron}

The identification of "problem nutrients" was not sensitive to the amount of breastmilk or iron RNI modelled. Thiamine was consistently the only "problem nutrient" identified for the young children. In contrast, the evaluation of FBRs was sensitive to the model parameters chosen. When the low breastmilk quantity was modelled, zinc and calcium no longer achieved $70 \%$ of their RNIs (57\% RNI for both calcium and zinc; Module III - worst case scenario analyses) for the best set of FBRs (i.e., VitAv14 - L7 - D14 - M7 - CG3). When high iron bioavailability was modelled, even though iron increased from $40 \%$ to $60 \%$ of its RNI, it remained below 70\% RNI for the same sets of FBRs modelled (shown in Supplemental

\section{Digital Tables 6 \& 7).}




\section{Discussion}

This study used LP to provide insights into how to adapt, for children aged 12-23m, existing food-based dietary guidelines, which were developed for 6-12 m old infants living in poor socioeconomic circumstances in Bogota, Colombia. The LP results indicate the adapted NCFg should include dairy and egg products in the set of recommendations to ensure a nutritionally adequate diet for young children aged 12-23 m. Promoting a varied diet that includes organ meat, fruits, vegetables, legumes, eggs and dairy products would ensure adequate intakes (at the population level) for all nutrients expect thiamine, niacin, folate and iron; whereas excluding dairy products or eggs from this set of recommendations would only ensure all children met their requirements for riboflavin and vitamins C, A and B12. The analyses also suggest it would be challenging to ensure population level dietary adequacy for all 11 micronutrients using local foods alone without the inclusion of fortified infant products to provide thiamin, niacin and iron. Niacin is unlikely to be of concern in practice given the conversion of tryptophan to niacin. A conservative approach was used in the selection of the iron RNI (i.e., assumed 10\% absorption), which may have under-estimated actual iron absorption when FBRs included daily servings of meat; especially given the RCT results showing increased haemoglobin levels in the NCFg intervention group (9). Red meat is an excellent food source of bioavailable iron (21). Nevertheless, when 15\% iron absorption was assumed, it was difficult to ensure dietary iron adequacy unless organ meat (4 servings/w) and vegetables (21 servings/w) were included. The criteria of $>70 \%$ of the RNI, however, is stringent for iron given the skewed iron requirement distribution for this age group (22).

LP is a powerful approach for objectively identifying nutrients that are likely to be low in local diets (i.e., problem nutrients). In this study, thiamine requirements were not achieved in any modelled diet unless fortified infant products were included in its food list. Thiamine deficiency has not been reported in Colombia, which suggests that children regularly consume 
thiamine fortified products, Alternatively, it is possible that thiamine values in the food composition database used were inaccurate or the thiamine status of these $12 \mathrm{~m}$ old children is marginal. Mild sub-clinical thiamine deficiency has been reported for Spanish parturient mothers and their newborns (23).

Similar to previous studies (24-27), our analyses highlight the importance of encouraging young children to consume foods from a range of food groups; which in this study included dairy products, meat, fish and eggs, legumes and vegetables. However, to ensure dietary adequacy, FBRs at the food sub-group level were needed, which in this case included milk, vitamin A-rich vegetables and chicken giblets. Young children have high nutrient requirements to support rapid linear growth (28-30); and likely need to consume the most nutrient dense foods within individual food groups to ensure their daily requirements for nutrients are met. The milk serving size modelled was $120 \mathrm{~g} / \mathrm{d}$; which needs emphasis if this recommendation is promoted, because high cows' milk consumption $(>500 \mathrm{ml} / \mathrm{d})$ is associated with an increased risk of anaemia $(31,32)$.

The LP analysis provided objective evidence for recommending organ meats in the NCFg to improve the nutritional quality of the diets of young children. This result is in line with previous studies showing that organ meat as an early complementary food is associated with improved zinc intake (33) and reduced risk of developing marginal iron status (4). It also showed the advantages of recommending 2 servings per day of vitamin A rich vegetables and a fortified infant product in the $\mathrm{NCFg}$; which both increases the number of nutrients for which population level dietary adequacy is achieved and potentially reduces the number of essential FBRs within the NFCg. Fortified foods such as commercial infant cereals and fortified flour (Bienesterina $\bigcirc$ ) were consumed by infants in this study (i.e., 12.5\% and 6.9\%, respectively); which suggests a recommendation to feed $12-23 \mathrm{~m}$ old children a fortified infant product once 
a day might be acceptable and affordable for inclusion in the NCFg. Acceptability trials, however, would need to be carried out to confirm this assumption.

The sensitivity analysis for BM intake showed that reducing the amount of BM modelled had a negative impact on dietary adequacy, in the absence of fortified infant products. The estimated breast milk intake of the infants in our study was similar to that reported by WHO (34). However, the breast feeding practices of infants enrolled in the RCT were not representative of the general population of Bogota, because one of the enrolment criteria was exclusive breastfeeding at 4 months of age. It is therefore likely that the BM consumption of 12-23 m old children in the general population is lower than that of our study population and that advice to continue breastfeeding until 2 y of age in the NCFg, is important for ensuring dietary adequacy for this population. This is in line with WHO/UNICEF infant and child recommendations $(2,3)$.

The most important limitations of this study were the sensitivity of LP to errors in the RNIs and the food composition database, especially for nutrients low in local diets. The analyses showed that $>70 \%$ of the RNIs for vitamin $\mathrm{C}$ and vitamin A were achieved without FBRs, which for vitamin A was surprising given evidence of vitamin A deficiency among infants in Colombia $(8,35)$. This discrepancy could occur if low maternal vitamin A status reduced the vitamin A content of breast milk below that of the breastmilk modelled (36). Another limitation is that FBRs might not be realistic for all children. Thus it is important to test them to ensure they are acceptable to the population. 


\section{Conclusion}

Optifood is a useful tool that can rapidly and objectively evaluate whether a proposed set of FBRs can ensure dietary adequacy of 11 micronutrients. This study shows how the NCFg would need to be modified for use in young children aged $12-23 \mathrm{~m}$ to ensure dietary adequacy for most children in the population. Modifying the NCFg to include vitamin-A rich vegetables (14 servings/w), dairy products (14 servings/w) including milk (7 servings/w), legumes (7 servings/w) and chicken giblets (3 servings/w), would likely ensure dietary adequacy for 8 of the 11 micronutrients. However, to ensure dietary adequacy for all 11 micronutrients, a recommendation for one serving per day of a fortified infant product would also have to be included in the NCFg. Ultimately, given the limitations of diet modelling, any modifications to the NCFg should be tested for acceptability, efficacy and safety by evaluating its rate of adoption and effects on biochemical status, growth and health outcomes. 


\section{References}

1. World Health Organization. Infant and young child feeding: model chapter for textbooks for medical students and allied health professionals. Geneva: WHO; 2009.

2. United Nations Children's Fund, World Health Organization, The World Bank. UNICEF-WHOWorld Bank Joint Child Malnutrition Estimates. New York: UNICEF, Geneva: WHO, Washington, DC: The World Bank; 2012.

3. World Health Organization, United Nations Children's Fund. Global strategy for infant and young child feeding. Geneva: WHO; 2003.

4. Dube K, Schwartz J, Mueller MJ, et al. Iron intake and iron status in breastfed infants during the first year of life. Clin Nutr 2010; 29(6):773-8.

5. Hambidge KM, Krebs NF, Sheng X, et al. Evaluation of meat as a first complementary food for breastfed infants: impact on iron intake \& growth. Nutr Rev. 2011; $69(0$ 1): 10.1111/j.17534887.2011.00434.x

6. The United Nations Children's Fund. The state of the world's children special edition. Statistical tables. New York: UNICEF; 2010.

7. The United Nations Children's Fund. State of the World's Children 2015 Country Statistical Information. New York: UNICEF; 2015.

8. Instituto Colombiano de Bienestar Familiar. Encuesta Nacional de la Situacion Nutricional en Colombia ENSIN [National Nutritional Survey]. Bogota: ICBF; 2010 (in Spanish).

9. Olaya GA, Lawson M, and Fewtrell MS. Efficacy and safety of new complementary feeding guidelines with an emphasis on red meat consumption: a randomized trial in Bogota, Colombia. Am J Clin Nutr. 2013; 98(4):983-93.

10. Olaya G.A, Borrero M.L. Propuesta para la formulación de pautas para la alimentación complementaria del niño lactante de 6 a 12 meses. Perspectivas En Nutrición Humana 2009; Vol. 11, Núm. 2 (in Spanish).

11. Ferguson EL, Darmon N, Briend A, et al. Food-based dietary guidelines can be developed and tested using linear programming analysis. J Nutr 2004; 134:951-7.

12. Ferguson EL, Darmon N, Fahmida U, et al. Design of optimal food-based complementary feeding recommendations and identification of key "problem nutrients" using goal programming. J Nutr 2006; 136: $2399-404$. 
13. Daelmans B, Ferguson E, Lutter CK, et al. Designing appropriate complementary feeding recommendations: tools for programmatic action. Matern Child Nutr 2013; 9:116-30.

14. Food and Agriculture Organization, World Health Organization. Human vitamin and minerals requirements. Reports of a joint FAO/WHO Expert Consultation. Rome: Food and Nutrition Division, Food and Agriculture Organization; 2002.

15. International Zinc Nutrition Consultative Group, Brown KH, Rivera JA, et al. International Zinc Nutrition Consultative Group (IZiNCG) technical document. Assessment of the risk of zinc deficiency in populations and options for its control. Food Nutr Bull 2004; 25:S99-203.

16. Lievano MEF., Parra K, Olaya G. Food data base. spreadsheet (Excel). 2008.

17. Dewey KG, Finley DA, Lonnerdal B. Breast milk volume and composition during late lactation (7-20 months). J Pediatr Gastroenterol Nutr, 1984. 3(5): p. 713-20.

18. Instituto Colombiano de Bienestar Familiar. Distribución, Cuidado, y uso de un Alimento de Alto Valor Nutricional, segunda edición. Bogota: ICBF; 2014 (in Spanish).

19. World Health Organization. Complementary feeding of young children in developing countries: a review of current scientific knowledge. Geneva: WHO; 1998.

20. Food and Agriculture Organization, World Health Organization, United Nations University. Human energy requirements. Report of a Joint FAO/WHO/UNU Expert Consultation. Rome: FAO, 2004.

21. Binnie MA, Barlow K, Johnson V, et al. Red meats: time for a paradigm shift in dietary advice. Meat Sci. 2014; 98(3):445-51.

22. World Health Organization, Food and Agricultural Organization of the United Nations. Guidelines on food fortification with micronutrients. Geneva: WHO, Rome: FAO; 2006.

23. Sánchez DJ, Murphy MM, Bosch-Sabater J, et al. Enzymic evaluation of thiamin, riboflavin and pyridoxine status of parturient mothers and their newborn infants in a Mediterranean area of Spain. Eur J Clin Nutr 1999; 53(1):27-38.

24. Ferguson EL, Darmon N, Fahmida U, et al. Design of optimal food-based complementary feeding recommendations and identification of key "problem nutrients" using goal programming. J Nutr 2006;136(9):2399-404.

25. Fahmida U, Santika O, Kolopaking R, et al. Complementary feeding recommendations based on locally available foods in Indonesia. Food Nutr Bull 2014; 35(4 Suppl):S174-9. 
26. Kolopaking R, Santika O, Sriani S, et al. Effectiveness in improving knowledge, practices, and intakes of "key problem nutrients" of a complementary feeding intervention developed by using linear programming: experience in Lombok, Indonesia. Am J Clin Nutr 2015;101(3):455-61.

27. Ferguson E, Chege P, Kimiywe J, et al. Zinc, iron and calcium are major limiting nutrients in the complementary diets of rural Kenyan children. Matern Child Nutr 2015;11 Suppl 3:6-20.

28. Dewey KG, Huffman SL. Maternal, infant, and young child nutrition: combining efforts to maximize impacts on child growth and micronutrient status. Food Nutr Bull. 2009;30:S187-9.

29. Victora CG, de Onis M, Hallal PC, et al. Worldwide timing of growth faltering: revisiting implications for interventions. Pediatrics 2010;125:e473-80.

30. Shrimpton R, Victora CG, de Onis M, et al. Worldwide timing of growth faltering: implications for nutritional interventions. Pediatrics 2001;107:E75.

31. Soh P, Ferguson EL, McKenzie JE, et al. Iron deficiency and risk factors for lower iron stores in 624-month-old New Zealanders. Eur J Clin Nutr. 2004;58(1):71-9.

32. Hopkins D, Emmett P, Steer C, et al. Infant feeding in the second 6 months of life related to iron status: an observational study. Arch Dis Child 2007;92(10):850-4.

33. Krebs NF, Westcott JE, Butler N, et al. Meat as a First Complementary Food for Breastfed Infants: Feasibility and Impact on Zinc Intake and Status. J Pediatr Gastroenterol Nutr 2006; 42 (2), 207-214. 2 34. Dewey KG, Brown KH. Update on technical issues concerning complementary feeding of young children in developing countries and implications for intervention programs. Food Nutr Bull. 2003;24(1):5-28.

35. Martínez-Torres J, Meneses-Echavéz JF, Ramírez-Vélez R. Prevalence and demographic factors associated with vitamin A deficiency in Colombian children aged 12-59 months. Endocrinol Nutr. 2014; 61(9):460-6.

36. Allen LH. Multiple micronutrients in pregnancy and lactation: an overview. Am J Clin Nutr. 2005; 81(5):1206S-1212S.

37. US Department of Agriculture, Agricultural Research Service, Nutrient Data Laboratory. USDA National Nutrient Database for Standard Reference, Release 27. Version Current: August 2014. Internet: http://www.ars.usda.gov/ba/bhnrc/ndl. Accessed July, 2014. 
38. Nutrient Data Laboratory, Beltsville Human Nutrition Research Center, Agricultural Research Service, US Department of Agriculture. USDA Table of Nutrient Retention Factors, Release 6. Maryland: Department of Agriculture; 2007. 


\section{Supplemental Digital Contents}

Supplemental Digital Table 1: Setting model parameters in Optifood and presentation of modules I to III

Supplemental Digital Table 2: Food serving size (g/day) and pattern descriptors (low, average and high constraints) for the $12-\mathrm{m}$ infants

Supplemental Digital Table 3: The nutrient content of modelled diets expressed as a percentage of Recommended Nutrient Intakes when all combinations of individual food based recommendation (FBR) from the NCFg and no recommendations are modelled

Supplemental Digital Table 4: The nutrient content of modelled diets expressed as a percentage of Recommended Nutrient Intakes for different scenarios of food-based recommendations (FBRs) impact without fortified food

Supplemental Digital Table 5: The nutrient content of modelled diets expressed as a percentage of Recommended Nutrient Intakes for different scenarios of food-based recommendations (FBRs) impact with fortified food

Supplemental Digital Table 6: The nutrient content of modelled diets expressed as a percentage of Recommended Nutrient Intakes for different combinations of individual food based recommendation (FBR) from the NCFg, different scenarios of FBRs with the greatest nutritional impact without fortified food assuming low breast milk intake

Supplemental Digital Table 7: The nutrient content of modelled diets expressed as a percentage of Recommended Nutrient Intakes when different combinations of individual food based recommendation (FBR) from the NCFg, different scenarios of FBRs with the greatest nutritional impact without fortified food assuming a high level of absorption for iron (15\% of bioavailability) 